\title{
Prospective evaluation of cerebral angiography and computed tomography in cerebral
} haematoma

\author{
S F S Halpin, J A Britton, J V Byrne, A Clifton, G Hart, A Moore
}

\begin{abstract}
One hundred and two consecutive patients with cerebral haematoma were prospectively allocated to one of two groups according to their CT on admission. Group 1 patients were thought to have a high probability of an underlying structural lesion and underwent cerebral angiography acutely. If normal, this was repeated at three months. Group 2 patients were thought not to have such a lesion and underwent angiography at three months. Patients older than the mean age of the study population, and hypertensive patients were much less likely to harbour an aneurysm or arteriovenous malformation than younger or normotensive patients ( $p<0.01$; sensitivity $87.9 \%$, specificity $88 \cdot 6 \%$ ). An aneurysm or arteriovenous malformation was the cause of haemorrhage in $12.8 \%$ of hypertensive patients, in $30.9 \%$ of patients with haematoma involving the basal ganglia, and $18 \cdot 2 \%$ of those with posterior fossa haemorrhage. Features of CT in isolation give a sensitivity of $77 \cdot 2 \%$ and a specificity of $84 \cdot 2 \%$. Follow up angiography in group 1 showed an arteriovenous malformation in one of seven patients in whom the original study was normal. These results contrast sharply with data from previous retrospective studies. The decision to investigate a patient with cerebral haematoma should be primarily based on the patient's clinical condition, rather than on the site of haemorrhage.
\end{abstract}

\section{$(\Im$ Neurol Neurosurg Psychiatry 1994;57:1180-1186)}

Stroke is caused by spontaneous cerebral haemorrhage in $4 \%$ to $11.9 \%$ of patients ${ }^{12}$ although rates of up to $40 \%$ have been quoted. ${ }^{3}$ Acute mortality ranges from $18 \%$ to $75 \%$, depending on the size and position of the haematoma and the age and clinical condition of the patient. ${ }^{145}$ To prevent recurrence, it is important to identify patients in whom haemorrhage is secondary to an underlying structural lesion, such as an aneurysm, vascular malformation, or tumour. Pathological studies have indicated that primary haemorrhage is most likely to occur in certain sites-in particular the basal ganglia and posterior fossa. ${ }^{6}$ Factors such as age, pre-existing systemic hypertension, and haematoma location are commonly employed in selecting patients for angiography. The influence of clinical features and CT on final diagnosis has been described retrospectively, ${ }^{7-11}$ but to our knowledge there has been no prospective angiographic assessment in life of the relative frequency of primary and secondary haemorrhage in patients with cerebral haematoma.

\section{Methods}

All patients presenting consecutively to this hospital between 1989 and 1992 with spontaneous cerebral haematoma were entered into the study. They were assigned to one of two groups after evaluation of their CT on admission by two or three consultant neuroradiologists. If the haematoma was thought likely to be due to an underlying structural cause, angiography was carried out as soon as the patient's condition allowed, and was repeated three months later if negative (group 1). If, however, such a lesion was not suspected, angiography was delayed by three months (group 2).

The radiologists were unaware of clinical details other than the patients' ages before assessing the admission CT. Clinical information was recorded for each patient after the CT assessment was made. Patients were declared hypertensive only if there was a documented history of systolic pressures greater than $140 \mathrm{~mm} \mathrm{Hg}$ or diastolic pressures greater than $90 \mathrm{~mm} \mathrm{Hg}$; if there was clinical evidence of longstanding systemic hypertension such as hypertensive retinopathy; or if such hypertension continued from admission to at least two weeks into the post-ictal period. The CT features used to assign patients to each group were the presence or absence of intraventricular or subarachnoid blood, calcification, or prominent vessels; and the site of haemorrhage. Contrast enhancement was not used in the initial assessment. Patients with predominantly subarachnoid haemorrhage were excluded from the study, as were those with a history of trauma. Angiography was carried out via the femoral artery, with selective injections made into those vessels appropriate to the potential blood supply of the site of the haematoma: most commonly this required ipsilateral internal, and sometimes external, carotid artery injections, and a vertebral arterial study. If an abnormality was found, the examination was extended as clinically appropriate.

Full informed consent was obtained from all patients before they were entered into the 
study, which was approved by the hospital ethics committee.

A $\chi^{2}$ test with Yates' correction was used for statistical analysis.

\section{Results}

One hundred and two patients entered the study (62 males, mean age 49.2 y (range $7-71$ ) years and 40 females, mean age $46 \cdot 4$ (range 10-70 years). Table 1 presents the

Table 1 Angiographic findings

\begin{aligned} & No of patients Diagnosis \\ & \hline 11 Aneurysms \\ & 31 Arteriovenous malformations \\ & 1 Glioma \\ & 1 Metastasis \\ & 38 No structural lesion found \\ & \hline\end{aligned}

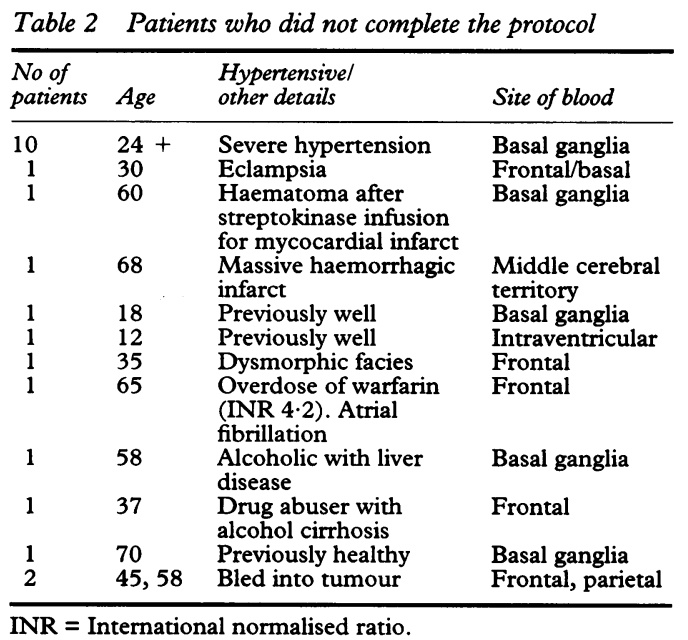

Table 3 Patients wrongly asigned to group 2

\begin{tabular}{|c|c|c|c|c|}
\hline $\begin{array}{l}\text { No of } \\
\text { patients }\end{array}$ & Age & $\begin{array}{l}\text { Clinical } \\
\text { details }\end{array}$ & Site of blood & Finding \\
\hline 2 & $50+$ & Hypertensive & \multirow{3}{*}{$\begin{array}{l}\text { Basal ganglia } \\
\text { or post fossa } \\
\text { Basal ganglia or } \\
\text { parieto-occipital } \\
\text { Basal ganglia/ } \\
\text { frontal }\end{array}$} & AVM \\
\hline 2 & $65+$ & Normotensive & & AVM \\
\hline 1 & 45 & $\begin{array}{l}\text { NHL with low } \\
\text { platelets on } \\
\text { chemotherapy }\end{array}$ & & PCom A \\
\hline 1 & 65 & $\begin{array}{l}\text { History of } \\
\text { DXT for glioma }\end{array}$ & \multirow{2}{*}{$\begin{array}{l}\text { Temporoparietal } \\
\text { Sylvian } \\
\text { Parietal }\end{array}$} & MCA \\
\hline 1 & 52 & $\begin{array}{l}\text { Presented one } \\
\text { month after } \\
\text { haemorrhage }\end{array}$ & & AVM \\
\hline 1 & 23 & Normotensive & \multirow{2}{*}{$\begin{array}{l}\text { Frontoparietal } \\
+ \text { IVH } \\
\text { Basal ganglia }\end{array}$} & AVM \\
\hline 1 & 66 & Normotensive & & $\begin{array}{l}\text { Cavernous } \\
\text { haemangioma }\end{array}$ \\
\hline
\end{tabular}

Positive angiographic findings in 10 patients whose initial CT did not suggest an underlying structural lesion. AVM $=$ arteriovenous malformation; $\mathrm{PCom} \mathrm{A}=$ posterior communicating
artery aneurysm; $\mathrm{NHL}=$ non-Hodgkin's lymphoma; $\mathrm{DXT}=$ radiotherapy; $\mathrm{MCA}=$ middle cerebral artery aneurysm; AVH = intraventricular haemorrhage.

Table 4 Influence of blood pressure on angiographic findings

\begin{tabular}{lllrll}
\hline & AVM & Aneurysm & Incomplete & Primary & Tumour \\
\hline Normotensive & $28(50 \cdot 1)$ & $8(14 \cdot 5)$ & $7(12 \cdot 7)$ & $10(18 \cdot 2)$ & $2(3 \cdot 6)$ \\
Hypertensive & $3(6 \cdot 4)$ & $3(6 \cdot 4)$ & $13(27 \cdot 7)$ & $28(59 \cdot 6)$ & 0
\end{tabular}

Number of haemorrhages due to structure and non-structural causes in 47 hypertensive, and 55 normotensive patients. Figures in parentheses are percentages of the subgroup. final diagnoses. Twenty two patients did not complete the full protocol, mostly because they were considered unfit for angiography or to undergo treatment should a causative lesion be found (12 patients). Four patients refused follow up angiography and four died without a postmortem during the study period. Two patients had an obvious tumour on initial CT (one oligodendroglioma and one metastasis); they did not have angiography, and are therefore considered as not having completed the protocol. Table 2 gives details of these 22 patients. Seven haematomas were evacuated before angiography. At operation, three arteriovenous malformations and one aneurysm were discovered; no causative lesion was found in the other three patients at surgery or at angiography three months later.

Thirty eight of 44 patients assigned to group 1 completed the protocol. Angiography showed causative arteriovenous malformations in 23 and aneurysms in nine. In one patient, CT showed an underlying tumour. Angiography was not performed and an oligodendroglioma was confirmed by biopsy. In another patient, with known melanoma, the haematoma was in association with several other lesions typical of metastases, and CT follow up confirmed a metastasis at the site of the haematoma. No abnormality was found in six patients, four of whom were aged under 35 and had frontal, frontotemporal, or temporoparietal haematomas. One 52 year old patient had a temporoparietal haematoma in association with subarachnoid blood, and one 48 year old hypertensive patient had a basal ganglia haemorrhage. Four patients in group 1 had an initial angiogram that showed only mass effect, and these patients failed to attend for further angiography.

Forty two of 58 patients assigned to group 2 completed the protocol. Angiography showed an arteriovenous malformation in eight and an aneurysm in two. No abnormality was found at delayed angiography in 32 . Table 3 presents the data for the 10 patients from group 2 in whom an angiographic structural lesion was found.

Table 4 details the findings in patients with normal and raised blood pressure. Forty seven per cent of all patients were hypertensive. There was a highly significant difference between the two $(p<0.001)$ relative to finding a structural lesion by chance.

The influence of age was assessed by separating the patients into two cohorts: those above and those below the mean age of the study population. There was a highly significant difference between the two age groups ( $p$ $<0.01$ ). Table 5 details the angiographic findings.

There was an even greater discrepancy ( $p<$ 0.01 ) when the results were compared between those who were hypertensive and older than the mean age of the study, and those who were normotensive and below the mean age (table 5). The combination of age and blood pressure in predicting the presence of a structural lesion at angiography gave a sensitivity of $87.9 \%$, and a specificity of 
Table 5 Influence of age and blood pressure on angiographic findings

\begin{tabular}{lll}
\hline & Structural lesion & No structural lesion \\
\hline Old patients $(\mathrm{n}=39)$ & $12(30 \cdot 8)$ & $27(69 \cdot 2)$ \\
Young patients $(\mathrm{n}=43)$ & $32(74 \cdot 4)$ & $11(25 \cdot 6)$ \\
Old hypertensive patients $(\mathrm{n}=35)$ & $4(11)$ & $31(89)$ \\
Young normotensive patients $(\mathrm{n}=35)$ & $29(83)$ & $6(17)$ \\
\hline
\end{tabular}

Angiographic findings in patients aged greater than the mean age of the study (old) and patients aged less than this (young) and the findings in hypertensive patients older than 47 and those in normotensive patients younger than 47 . Figures in parentheses are the percentage of the subgroup.

Table 6 Influence of site of blood on angiographic findings

\begin{tabular}{lclcc}
\hline & AVM & Aneurysm & Incomplete & No lesion \\
\hline Frontal (n= 23) & $7(35)$ & $6(30)$ & $4(20)$ & $6(30)$ \\
Temporal (n=34) & $14(41 \cdot 2)$ & $7(20 \cdot 6)$ & $1(2 \cdot 9)$ & $12(35 \cdot 3)$ \\
Parietal (n=31) & $10(32 \cdot 3)$ & $3(9 \cdot 7)$ & $4(12 \cdot 4)$ & $14(45 \cdot 2)$ \\
Occipital (n=6) & $1(16 \cdot 7)$ & 0 & $1(16 \cdot 7)$ & $4(66 \cdot 7)$ \\
Basal ganglia (n=26) & $6(23 \cdot 1)$ & $2(7 \cdot 8)$ & $10(38 \cdot 4)$ & $8(30 \cdot 1)$ \\
Post fossa (n=11) & $2(18 \cdot 2)$ & 0 & $4(36 \cdot 4)$ & $5(45 \cdot 5)$ \\
Sylvian (n = 14) & $6(43)$ & $6(43)$ & $1(7)$ & $1(7)$ \\
Ventricles (n=3) & $2(66)$ & 0 & $1(33)$ & 0 \\
IVH (n=38) & $11(29)$ & $4(11)$ & $9(24)$ & $14(3637)$ \\
IVH + SAH (n=8) & $2(25)$ & $3(37)$ & $1(12)$ & $2(25)$ \\
IVH-SAH (n=30) & $9(30)$ & $1(3)$ & $8(26)$ & $12(40)$ \\
SAV-IVH (n=11) & $7(64)$ & $2(18)$ & 0 & $2(18)$ \\
\hline
\end{tabular}

Angiographic findings when at least part of the haemorrhage occupied the specified site. Figures in parentheses are the percentages for that subgroup. Basal ganglia = any combination
of caudate, globus pallidus, putamen, thalamus, internal or external capsule, or insula; IVH = of caudate, globus pallidus, putamen, thalamus, internal or external capsule, or insula; IV
intraventricular haemorrhage; SAH = subarachnoid haemorrhage; post fossa = brainstem and/or cerebellum; sylvian = a haematoma that bridges the sylvian fissure.

Figure 125 year old patient with sudden onset of right hemiparesis and obtunded consciousness. (A) Non-contrast axial CT scan shows

haematoma in the posterior left frontal lobe with some surrounding low density. The patient was allocated to group 1. (B) Late arterial/capillary phase of left internal carotid arteriogram performed the day after the haemorrhage. There is an avascular area (arrows) but no other evidence to suggest an arteriovenous

malformation. (C) Lateral non-contrasted $T 1$ weighted MRI (SE $T R / T E=600 / 15) . A$ small focus of high signal was interpreted as representing residual haematoma. An arteriovenous malformation was not demonstrated. (D) Arteriogram performed at three months. There is a small leash of abnormal vessels high in the left frontal lobe with early venous drainage into the sagittal sinus (arrows).

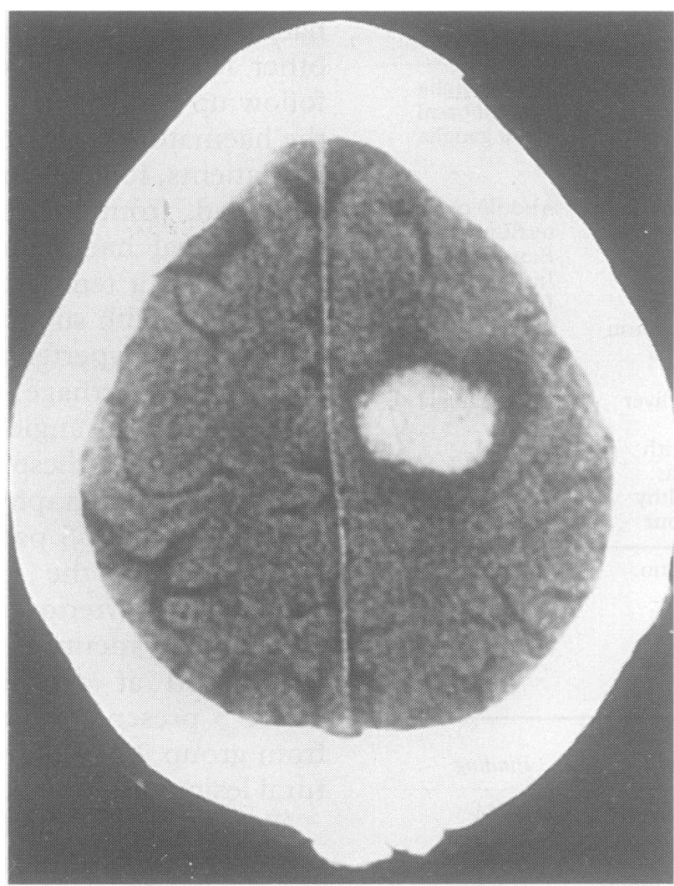

A

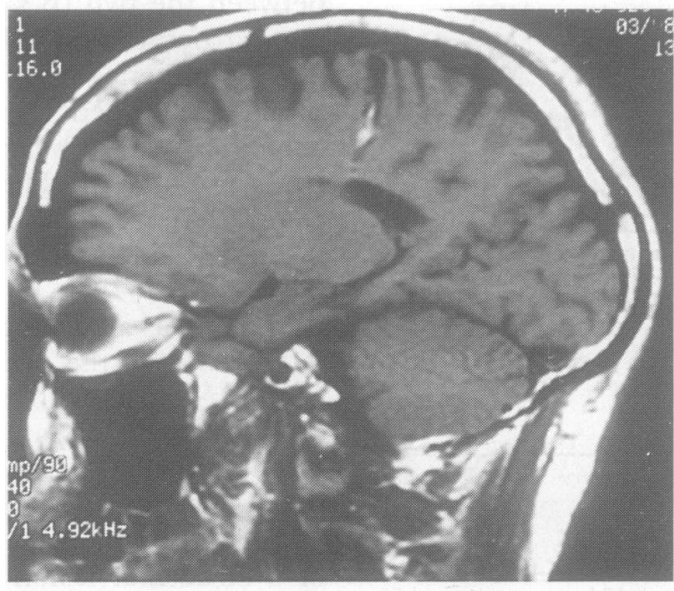

C
$88.6 \%$, whereas CT evaluation in isolation from clinical data had a sensitivity of $77 \cdot 2 \%$ and a specificity of $84.2 \%$ in predicting an underlying lesion by radiology.

The location of the haematoma and the presence or absence of free subarachnoid blood or intraventricular blood also influenced the chance of finding a structural lesion at angiography (table 6). Although there was a trend for a haematoma in the frontal or temporal lobe to be associated with an arteriovenous malformation or aneurysm, but for no structural lesion to be found with a parietal or occipital haematoma, this did not reach statistical significance. A haematoma crossing the sylvian fissure is likely linked with a structural cause. The combination of a haematoma with subarachnoid haemorrhage but no intraventricular blood is also linked with a structural cause.

Nearly $13 \%$ of hypertensive patients had an underlying arteriovenous malformation or aneurysm, (this may be an underestimate, as several hypertensive patients did not complete the protocol). A structural lesion was found in $30.9 \%$ of patients with basal ganglia haemorrhage, and $18.2 \%$ of those with posterior fossa haemorrhage. Of five pure putamenal haematomas, no cause was found in four, but in one, a venous anomaly was seen on angiography and subsequent MRI showed a cavernous haemangioma.

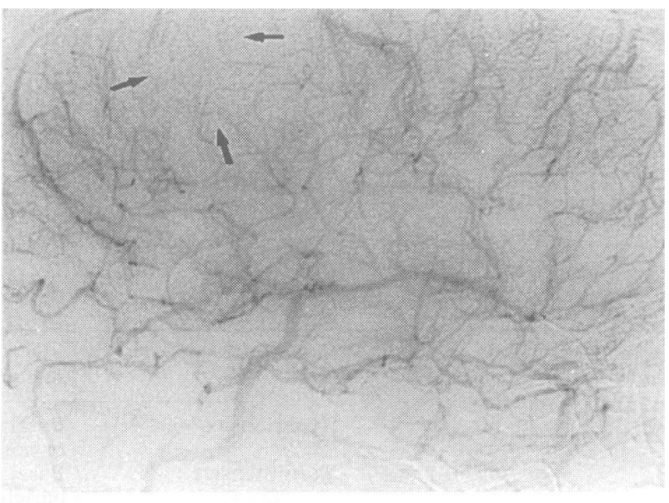

B

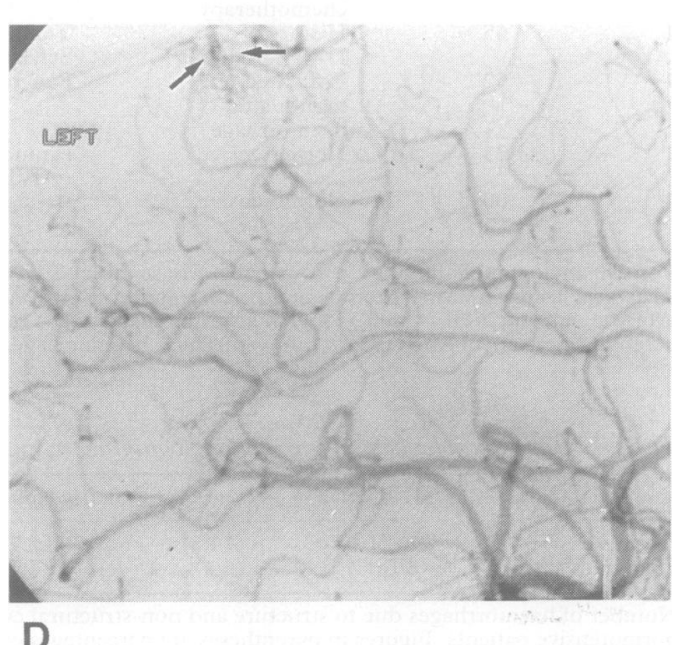


Figure 2 A 66 year old hypertensive man with sudden onset of hemiparesis. (A) CT shows a small haematoma in the left thalamus. There is patchy low density change in the cerebral white matter seen on this and other axial images suggesting small vessel disease. This patient was allocated to group 2 . (B) Early arterial phase lateral left vertebral arteriogram performed three months after ictus. There is an arteriovenous

malformation supplied by posterior cerebral arteries with early venous drainage into the vein of Galen. This patient was treated successfully by radiosurgery.

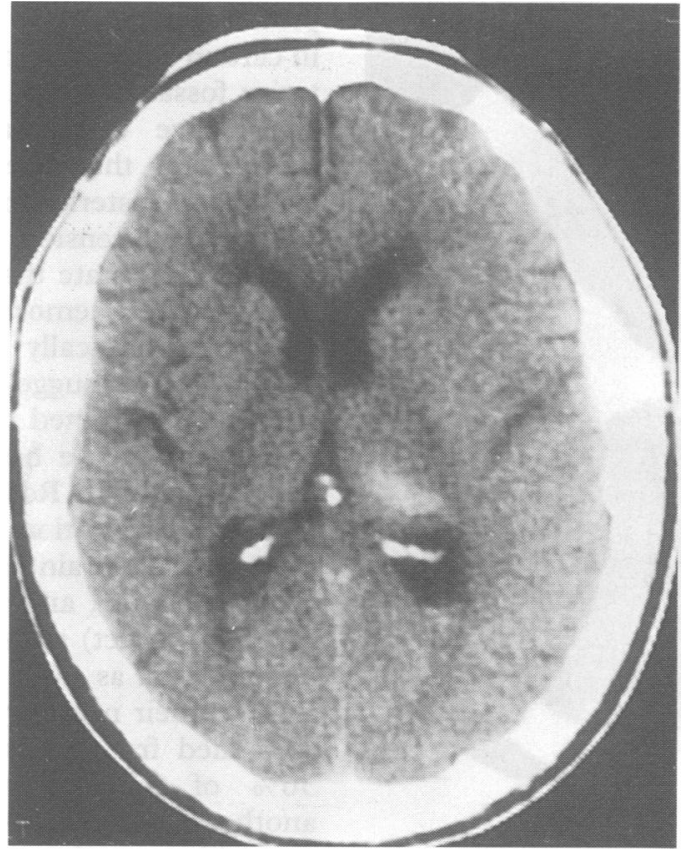

A

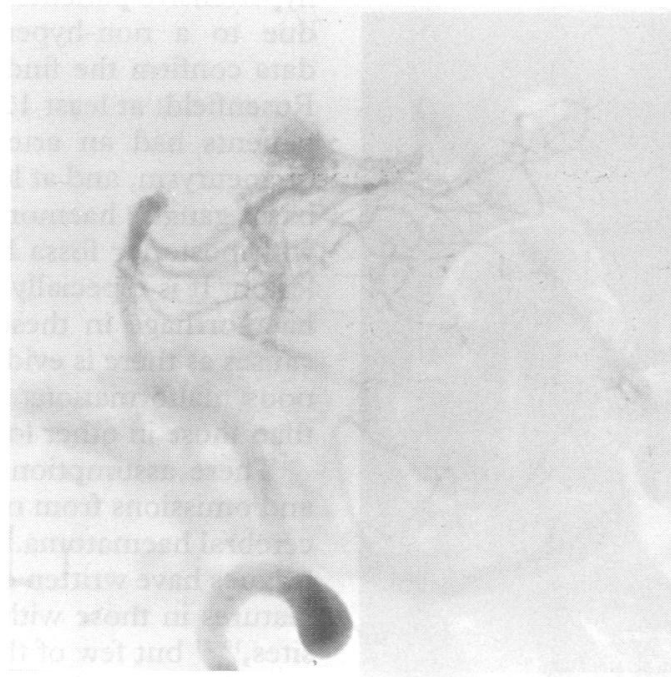

B

Seven patients in group 1 had angiography repeated at three months. These studies identified one arteriovenous malformation, and in three patients showed prominent small veins that were attributed to haemorrhage or surgery performed to evacuate the haematoma.

Computed tomography showed only intraventricular haemorrhage in three patients, each of whom was normotensive and aged under 40. Two had a choroidal arteriovenous malformation, and in one no angiographic abnormality was found. Further angiography was refused by this child's parents, but subsequent MRI showed no evidence of vascular abnormality.

Three incidental aneurysms were detected during angiography in two patients, remote from the site of haemorrhage. These patients have been included in the "non-structural" group.

There was only one angiographic complication: a transient dysphasia in a 48 year old

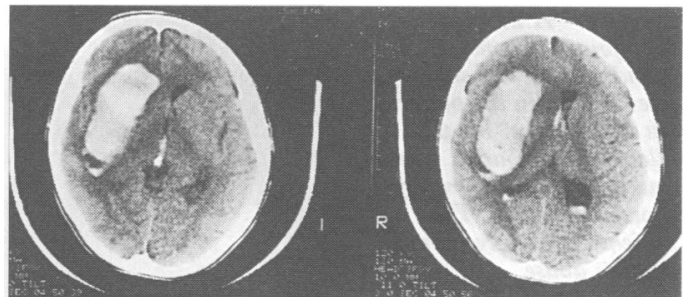

A

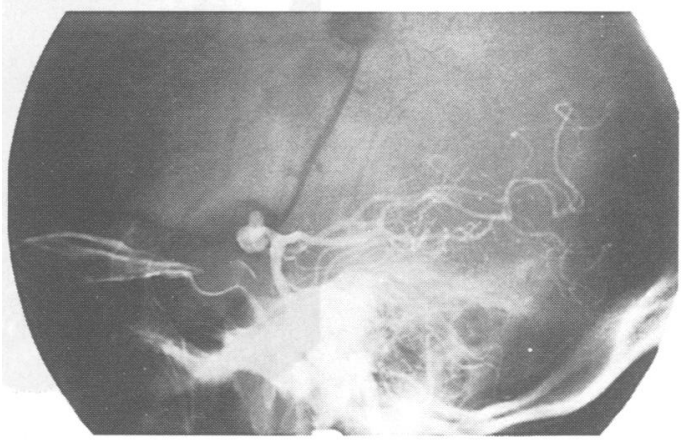

B

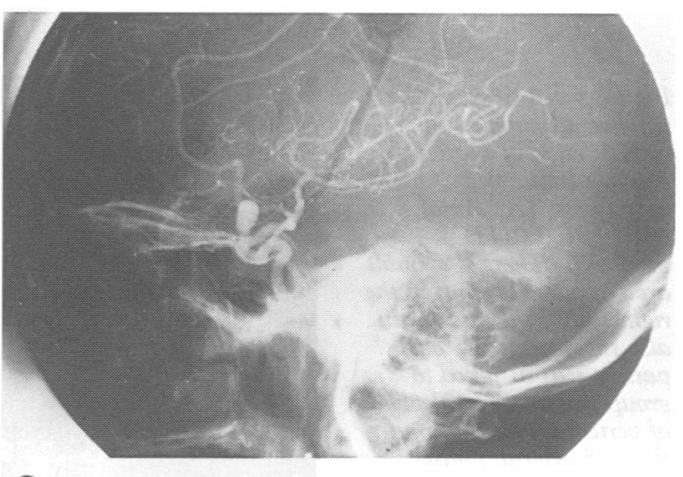

C

Figure 3 A 38 year old woman admitted after collapse and loss of consciousness. She was thrombocytopaenic after chemotherapy for non-Hodgkin's lymphoma. (A) Contiguous axial $C T$ shows haematoma in the right basal ganglia, with a moderate mass effect. The patient was allocated to group 2. Lateral views of vertebral (B) and right carotid $(C)$ arteriograms were taken three months after a craniotomy to evacuate the haematoma. There was an aneurysm at the level of the ophthalmic artery and another at the junction of the right posterior cerebral artery and posterior communicating artery. At surgery the second aneurysm was found to have bled.

hypertensive patient with a frontal haematoma.

Representative scans and angiograms are shown in figures $1-5$.

\section{Discussion}

The first successful evacuation of a cerebral haematoma was carried out in 1888 by McEwan, ${ }^{12}$ whose patient made a complete recovery from an upper limb monoplegia. McKissock and coworkers, at this hospital reported in 1959 that the best predictor of outcome after surgery for this condition was the preoperative condition of the patient. ${ }^{1314}$ After successful treatment of acute cerebral haemorrhage, further management depends on the risk of further haemorrhage. How this risk should be assessed becomes critical Russell's paper in $1953^{\circ}$ indicated that 
Figure 4 A 23 year old man found unconscious by friends. Axial CT shows haematoma in the posterior left frontal lobe. He was allocated to group 1. Early and delayed angiography showed no lesion. The patient eventually admitted cocaine abuse.

Figure 5 A 56 year old woman admitted with sudden onset of headache, double vision, and drowsiness. (A) Axial $M R I$ (SE TR/TE = 2500/95). There is a small area of altered signal in the right side of the mid-brain, also shown on $C T$. This patient was assigned to group 2. (B) Lateral view of vertebral arteriogram in the mid-arterial phase performed three months after ictus. There is a small arteriovenous malformation (arrows) with early venous drainage into the straight sinus.

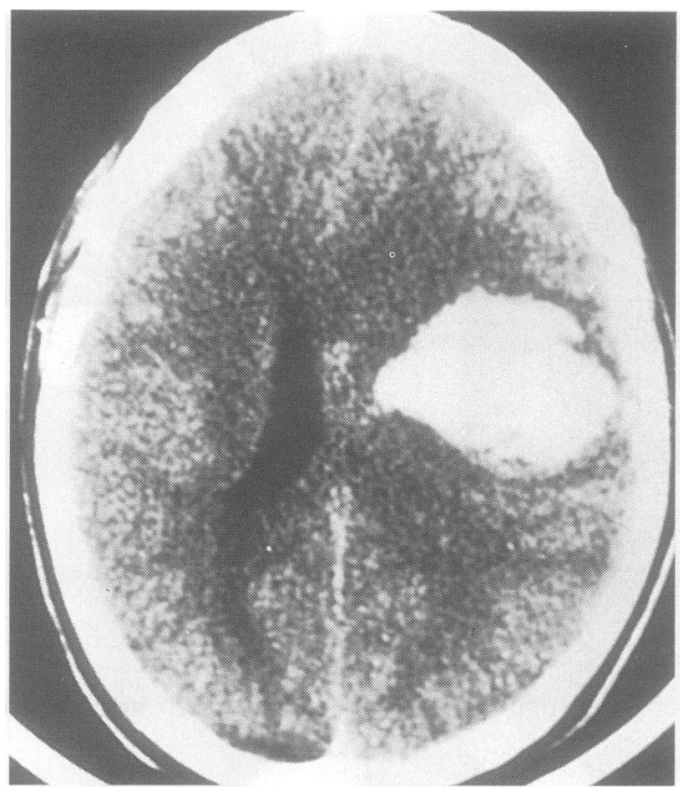

primary haemorrhage was most likely to occur in certain sites-notably the striatum and posterior fossa. It would seem that many physicians have extrapolated this to the false assumption that haemorrhage in the basal ganglia or posterior fossa is almost exclusively due to hypertension. Douglas and Haerer, ${ }^{15}$ for example, state that when the diagnosis of hypertensive haemorrhage is obvious, angiography is "ethically unacceptable", whereas others have suggested that angiography should be restricted to patients with haemorrhage outside the basal ganglia. ${ }^{16}{ }^{17}$ This led McCormick and Rosenfield in 1973 to state that "the assumption that hypertension causes most massive brain haemorrhage has become virtual dogma", and "the majority of reports (on this subject) are so poorly controlled and documented as to be of little scientific validity". In their necropsy review of 144 patients who died from massive brain haemorrhage, $36 \%$ of patients with hypertension had another cause of haemorrhage, a third having causative arteriovenous malformation or aneurysms; and 19 normotensive and five hypertensive patients had striatal haemorrhage due to a non-hypertensive aetiology. ${ }^{4}$ Our data confirm the findings of McCormick and Rosenfield: at least $12 \cdot 8 \%$ of our hypertensive patients had an arteriovenous malformation or aneurysm, and at least $31 \%$ of patients with basal ganglia haemorrhage and $18 \%$ of those with posterior fossa haemorrhage had such a lesion. It is especially important not to ascribe haemorrhage in these sites to non-structural causes as there is evidence that such arteriovenous malformations are more likely to bleed than those in other locations. ${ }^{18}$

These assumptions have led to exclusions and omissions from many studies dealing with cerebral haematoma..$^{7-11}{ }^{19-21}$ Weisberg and colleagues have written extensively on the clinical features in those with haematomas in varying sites, ${ }^{19-21}$ but few of the patients had angiography. Loes et $a l^{9}$ reviewed the records and radiology of 67 patients referred for angiography after cerebral haemorrhage. In addition to this initial selection, patients with posterior fossa, basal ganglia, or thalamic haemorrhage were excluded, as were patients with bleeding diathesis. Such selection may exclude patients liable to rebleed from structural lesions. In our study three patients had haemorrhage during warfarin treatment, all with an international normalised ratio of greater than 3.5 , one of whom was found to have an unsuspected arteriovenous malformation on angiography, and one patient with thrombocytopenia after chemotherapy for non-Hodgkin's lymphoma bled from an aneurysm. Tofol $e t$ al retrospectively reviewed two groups of patients with non-traumatic intracerebral haemorrhage, ${ }^{10}{ }^{11}$ without significant exclusions. They found a positive angiography rate of $12.8 \%$ in hypertensive patients, exactly matching our figure, but concluded that "most hypertensive patients, in particular those with putamenal haemorrhages, do not require arteriography".

The sensitivity and specificity of CT in 
predicting an angiographic abnormality seem disappointing in this study when compared with others, ${ }^{7-10}$ who have found that CT is accurate in predicting the presence of an underlying structural lesion. These authors have used entry criteria less broad than ours. We included patients with basal ganglia or posterior fossa haemorrhage, tending to place them in group 2 according to available literature, and were proved wrong on several occasions. Hayward and O'Reilly, ${ }^{8}$ using a first generation CT scanner with a $160 \times 160$ matrix, reported that $\mathrm{CT}$ is $90 \%$ accurate in predicting the cause of cerebral haemorrhage, but excluded all patients with posterior fossa haemorrhage, those below the age of 21 , and patients on whom angiography was not performed. More recently, in a retrospective study of 149 patients with cerebral haematoma, Laissy et al reported sensitivity and specificity of CT to be $96 \%$ and $100 \%$ respectively in detecting arteriovenous malformation or aneurysm, using a combination of CT findings, including site of haemorrhage and the presence or absence of intraventricular or subarachnoid haemorrhage. ${ }^{7}$ Their 149 patients were selected from a total of 452 , however, and no information is given as to how patients were selected for angiography. Similar to McCormick et al, they found that neither age nor systemic blood pressure were good predictors of patients with abnormal angiograms.

There were fewer aneurysms in our study population compared with other studies. ${ }^{7910}$ We attributed this to the fact that we excluded patients whose scans predominantly showed subarachnoid haemorrhage.

Repeating angiography when an acute study does not show a structural lesion has been advocated by Willinsky et $a l,{ }^{22}$ who state that up to $15 \%$ of small arteriovenous malformations were not shown on the first examination, presumably due to a mass effect from haematoma. We discovered one arteriovenous malformation at follow up angiography, which had not been previously apparent, and Laissy et al found three "angiomas" in this manner. ${ }^{7}$ An alternative approach, used in our group 2, is not to perform angiography acutely in those patients for whom surgery is not contemplated, but to wait until any mass effect had subsided. We discovered an arteriovenous malformation or aneurysm in 10 such patients, none of whom came to harm by the delay in diagnosis. By the same token, 48 patients were saved from having two rather than one angiogram. We would accept, however, that delay in diagnosing and treating an aneurysm may have serious consequences.

Like us, Loes et $a l^{9}$ found an association between location of haematoma and angiographic abnormality, and speculate that the small proportion of structural lesions found with haematoma in the parietal and occipital lobes may be related to the predilection of amyloid angiopathy for these areas. ${ }^{23}$ This diagnosis may be difficult to make, and its occurrence might contribute to the small numbers of positive angiograms in elderly patients.
Of course, there may be many diagnoses hidden within a diagnosis of "primary" haemorrhage: lesions such as cavernous haemangioma and vasculitis, for example, are unlikely to be shown by angiography. ${ }^{24} 25$ Other conditions predisposing to cerebral haemorrhage such as bleeding diatheses, diabetes mellitus, and liver disease ${ }^{426-28}$ should be detectable by clinical and laboratory investigation. A history of drug abuse may be difficult to elicit, but can cause cerebral haemorrhage, ${ }^{2930}$ and haemorrhage into a tumour will sometimes not reveal the underlying cause until the haematoma has absorbed. ${ }^{31}{ }^{32}$ In our experience this is unusual, however.

Although this study collected consecutive patients prospectively, they were a selected group by referral to our hospital. Therefore patients who were considered too sick to be transferred and those dying before arrival were excluded. Other logistic factors may have biased the series, which might explain the paucity of patients with tumours, as such patients may not have been sent to us. Similarly, elderly patients, in whom the presumed diagnosis was of a non-structural lesion, may not have been referred.

Such selection, although unintentional on our part, may have biased our results by increasing the percentage of patients in whom a structural lesion was found. We have no measure of how many patients with cerebral haemorrhage are not referred because the clinician believes they have a "primary" bleed, nor how such a clinician makes this decision. Similarly, we do not know how often the diagnosis of haemorrhage is not made because the patient presenting with a stroke does not have CT. As the mean age of our study group was 46.4 (range 7-71) years, we suspect that many elderly patients who are likely to have amyloid angiopathy were not referred.

We used angiography as our gold standard and it is questionable whether MRI or magnetic resonance angiography (MRA) would be preferable. Whereas MRI may throw further light on the cause of haemorrhage within the "primary" group, there is as yet no evidence to suggest that it would be as sensitive as angiography in the detection of arteriovenous malformation. ${ }^{33}$ Willinsky et al state that very small arteriovenous malformations are, by their nature, occult on MRI. ${ }^{22}$ In one of our patients, MRI did not detect a small arteriovenous malformation, but MRA was not performed. A prospective comparison between MRI, MRA, and angiography in patients with cerebral haematoma is currently under assessment at this hospital.

In summary, we believe our data support the recommendation that angiography should be considered in all patients with cerebral haematoma, regardless of the site of haemorrhage, or a history of hypertension, and that the clinical condition of the patient should be the deciding factor. We further believe that an angiogram that shows no abnormality apart from mass effect should be repeated after an appropriate interval, especially if the patient is young and normotensive. 
1 Waltimo O, Kaste M, Aho K, Kotila M. Outcome of stroke in the Espoo-Kauniainen area, Finland. Ann Clin Res 1980;12:326-30.

2 Tsemetis SA. Surgical mangement of intracerebral haematomas. Neurosurgery 1985;16:562-72.

3 Jellinger K. Intracranial aneurysms: pathology and aetiology. In: Pia HW, ed. Intracranial aneurysms. Berlin Springer, 1979:5-19.

4 McCormick WF, Rosenfield DB. Massive brain haemorrhage: a review of 144 cases and an examination of their causes. Stroke 1973;4:946-54.

5 Sahs AL, Perrett GE, Locksley HB. Intracranial aneurysms and subarachnoid haemorrhage: a cooperative study. Philadelphia: Lippincott and Co, 1969.

6 Russell DS in discussion: The pathology of spontaneous intracranial haemorrhage. Proceedings of the Royal Societ of Medicine 1953;47:689-704.

7 Laissy JP, Normand G, Monroe M, Duchateau C, Aliber $A$, Thiebot J. Spontaneous intracerebral haematomas from various causes. Predictive value of CT compared with Angiography. Neuroradiology 1991;33:291-5.

8 Hayward RS, O'Reilly GVA. Intracerebral Haemorrhage. Accuracy of computerised axial scanning in predicting the underlying pathology. Lancet 1976;i:1-4.

9 Loes DJ, Smoker WRK, Biller J, Cornell SH. Non traumatic lobar intracerebral haemorrhage: CT/Angiographic correlation. American fournal of Neuroradiology 1987;8:1027-30.

10 Toffol GJ, Biller J, Adams HP, Smoker WR. The predicted value of arteriography in nontraumatic intracerebral haemorrhage Stroke 1986;17:881-3.

11 Tofol GJ, Biller J, Adams HP Jr. Nontraumatic intracerebral haemorrhage in young adults. Arch Neurol 1987;44: 483-5.

12 MacEwan W. An address on the surgery of the brain and spinal cord. $B M \mathcal{F} 1888 ; 2: 302-9$.

13 McKissock W, Richardson A, Walsh I. Primary intracerebral haemorrhage: results of the surgical treatment of 214 consecutive cases. Lancet 1959;ii:683-6.

14 McKissock W, Richardson A, Taylor J. Primary intracerebral haemorrhage: a controlled trial of surgical and conservative treatment in 180 unselected cases, Lancet $1961 ; \mathbf{i i}: 221-6$.

15 Douglas MA, Haerer AF. Long term prognosis of hypertensive intracerebral haemorrhage. Stroke 1982;13: 488-91.

16 Papo I, Janny P, Caruselli G, Colnet G, Luongo A Intracranial time pressure course in primary intracerebral haemorrhage. Neurosurgery 1979;4:504-11.
17 Ojeman RG, Mohr JP. Hypertensive brain haemorrhage. Clin Neurosurg 1976;23:220-4

18 Willinsky $R$, Lasjaunias $P$, Terbrugge $K$, Pruvost $P$ Malformations arterioveineuses cerebrales. Analyse de l'angio-archictecture chez des patients ayant presente un accident hemorragigue. $\mathcal{f}$ Neuroradiol 1988;15 225-37.

19 Weisberg LA. Subcortical lobar intracerebral haemorrhage: clinical computed tomographic correlations. I Neurol Neurosurg Psychiatry 1985;48:1078-84.

20 Weisberg LA, Stazio A, Shamsnia M, Elliott D. Nontraumatic temporal subcortical haemorrhage, clinical computed tomographic analysis. Neuroradiology 1990;32:127-41.

21 Weisberg LA. CT in intracranial haemorrhage. Arch Neurol 1979;36:422-42.

22 Willinsky R, Lasjaunias P, Comoy J, Provost P. Cerebral micro arteriovenous malformations. Review of 13 cases. Acta Neurochir (Wien) 1988;91:37-4

23 Vinters HV, Gilbert HJ. Cerebral amyloid angiopathy II, the distribution of amyloid vascular changes. Stroke 1983;14:924-8.

24 Bitoh S, Hasegawa H, Fujiwara $M$, Sakurai $M$. Angiographically occult vascular malformations causing
intracranial haemorrhage. Surg Neurol 1982;17:435-42.

25 Edwards KR. Haemorrhagic complications of cerebra arteritis. Surg Neurol 1988;17:137-40.

26 Ojeman RG, Heros RC. Spontaneous brain haemorrhage. Stroke 1983;14:468-75.

27 Almaani WS, Awidi AS. Spontaneous intracranial bleeding in haemorrhagic diathesis. Surg Neurol 1982;17: in haer $137-40$.

28 Ruscadella J, Peiro A. Prognostic factors in intraparenchymatous haematoma with ventricular haemorrhage. Neuroradiology 1986;28:34-7.

29 Yu YJ, Cooper DR, Wellenstein DE, Block B. Cerebral angiitis and intracerebral haemorrhage associated with metamphetamine abuse. $\mathcal{F}$ Neurosurg $1983 ; 58: 109-11$.

30 Bessen HA. Intracranial haemorrhage associated with phenylcyclidine abuse. $\mathcal{F A M A} 1982 ; 248: 585-6$.

31 Scott $M$. Spontaneous intracerebral haematoma caused by cerebral neoplasms. F Neurosurg 1975;42:338-42.

32 Gildersleeve N, Koo AH, McDonald CJ. Metastatic tumour presenting as intracerebral haemorrhage. Radiology 1977;124:109-12.

33 Willinsky RA, Fitzgerald $M$, Terbrugge $K$, Montanera W, Wallace $M$. Delayed angiography in the investigation of intracerebral haematomas caused by small arteriovenous malformations. Neurology 1993;35:307-11. 